\title{
Effectiveness and cost-effectiveness of height- screening programmes during the primary school years: a systematic review
}

\author{
D Fayter, ${ }^{1} \mathrm{~J}$ Nixon, ${ }^{1}$ S Hartley, ${ }^{2}$ A Rithalia, ${ }^{1}$ G Butler, ${ }^{3}$ M Rudolf, ${ }^{4}$ P Glasziou, ${ }^{5}$ M Bland ${ }^{6}$ \\ L Stirk, ${ }^{1}$ M Westwood ${ }^{1}$
}

\section{See Perspective, p 267}

- Additional tables are published online only at http:// adc.bmi.com/content/vol93/ issue4

${ }^{1}$ Centre for Reviews and Dissemination, University of York, York, UK; ${ }^{2}$ Clinical Trials Research Unit, University of Leeds, 17 Springfield Mount, Leeds, UK; ${ }^{3}$ Institute of Health Sciences, University of Reading, London Road, Reading, UK;

${ }^{4}$ Department of Community Paediatrics, University of Leeds, Belmont House, 3-5 Belmont Grove, Leeds, UK; ${ }^{5}$ Department for Primary Health Care, University of Oxford, Old Road Headington, Oxford, UK

${ }^{6}$ Department of Health

Sciences, University of York, Heslington, York, UK

Correspondence to: Dr Marie Westwood, Senior Research Fellow, Centre for Reviews and Dissemination, University of York, York, Y010 5DD; mew3@york.ac.uk

Accepted 25 April 2007 Published Online First 2 May 2007

\section{ABSTRACT}

Objective: To determine the effectiveness and costeffectiveness of height screening (of children aged 4 to 11) to identify height-related conditions.

Design: Systematic review and economic modelling. Setting and intervention: We included published and unpublished screening studies of any design, except case reports, conducted in any setting that measured children's height as part of a population-level assessment. Studies were identified by electronic database searches, contact with experts and from bibliographies of retrieved studies. Participants: Children aged between 4 and 11 years. Outcome measures: Diagnostic yield of height-related conditions and change in quality of life, as measured by quality-adjusted life years (OALYS), for early versus late treatment of underlying conditions.

Results: Twelve studies described a height-screening programme and provided data on the diagnostic yield of newly diagnosed height-related conditions. Where reported, yield for growth-hormone deficiency (per 1000 children screened) ranged from 0.05 (1 in 20 000) to 0.62 (approximately 1 in 1500) and for Turner syndrome (per 1000 children screened) was between 0.02 (1 in 50 000) and 0.07 (approximately 1 in 14 000). As a secondary gain, children with other potentially treatable conditions were identified; diagnostic yields ranged from 0.22 to 1.84 per 1000 children screened. Three studies did not detect any new cases, but all of these studies had methodological limitations. Economic modelling suggested that height screening is associated with health improvements and is cost effective for a willingness to pay threshold of $£ 30000$ per QALY.

Conclusions: This review indicates the utility and acceptable cost-effectiveness of height screening arising from increased detection of height-related disorders and secondary pick-up of other undiagnosed conditions.

Further research is needed to obtain more reliable data on quality of life gains and costs associated with early interventions for height-related conditions. The exact role of height-screening programmes in improving child health remains to be determined.

A child's height and weight is well established as an indicator of general health and well-being. Assessment of height and weight can lead to the identification of treatable disorders in the apparently normal child. Despite this, children with a treatable cause of abnormal growth are frequently diagnosed at a late age or treatment is initiated at a late stage. ${ }^{1-5}$ The role of height-screening programmes for primary-school-aged children in identifying height-related disorders is currently unclear and uncertainties exist about the most appropriate age(s) at which to measure and the measurement strategies to adopt to minimise late referrals.

Regular screening of children's height does not aim to detect a single pathology. There are a number of conditions that may lead to decreased or increased growth rate and/or short or tall stature. Conditions in which stature outside the normal range is often the only or most significant presenting feature are growth-hormone deficiency (GHD) and Turner syndrome (TS), and it is these conditions that are used to justify height screening. ${ }^{6}$ However, new cases of a number of other conditions may be identified as a consequence of height screening. Short stature may result from hypothyroidism, psychosocial deprivation, intrauterine growth retardation or other chronic illness. It many instances, however, short stature has no underlying pathology and may be genetic or have no obvious explanation (idiopathic short stature or ISS). Tall stature is a feature of a number of syndromes (eg, Marfan and Klinefelter) and may also indicate treatable endocrine disorders. Early detection and diagnosis of organic causes of abnormal growth is important to optimise final adult height and minimise the health impact of any underlying condition. ${ }^{2-9}$ Where possible, treatment will be provided for the underlying condition. In cases where the condition is not treatable, early diagnosis can allow for discussion with the family and child, and counselling can be initiated to minimise adverse psychological effects.

There is no standard "cut-off" used for defining short or tall stature. Diagnosis of abnormal growth is usually based on a child's height measurement outlying recommended percentile points on a population-specific growth chart. ${ }^{10}$ In the UK, a height below the 0.4th centile of the UK 1990 charts has been recommended to define short stature and a height above the 99.6th centile to define tall stature in need of further investigation. ${ }^{6}$ The Child Health Subcommittee of the UK National Screening Committee recommended in 2004 that a single height and weight measurement should be taken at or around the time of school entry and that the 0.4 th centile for height should be used to initiate referral. ${ }^{11}$ However, historically, routine growth-screening practices have varied across the $\mathrm{UK}^{12}$, and adherence to current guidance is unknown. Looking outside the UK, variations in practice also exist between countries. ${ }^{13} 14$ 


\section{METHODS}

Our objective was to perform a systematic review of the effectiveness and economic modelling of height screening in primary-school-aged children (4 to 11 years) to identify heightrelated conditions. The review also included screening for obesity, but this paper focuses on stature only.

\section{Effectiveness review}

The systematic review was undertaken in accordance with the Centre for Reviews and Dissemination (CRD) guidelines. ${ }^{15} \mathrm{We}$ accepted studies of any design with the exception of case reports. To allow direct relevance to the UK population, studies had to include children aged between 4 and 11 years in Western Europe, North America or Australia/New Zealand (excluding studies of aboriginal populations). Target conditions were GHD, TS, juvenile hypothyroidism (JH), psychosocial growth failure and clinical conditions associated with tall stature (including precocious sexual maturation, Klinefelter and Marfan syndromes). Studies had to measure children's height as part of a population-level assessment. Accepted outcomes measures were the diagnostic accuracy of height screening (sensitivity and specificity), "diagnostic yield" of height-related conditions, age at diagnosis, route to diagnosis and patientmanagement outcomes such as referral.

Studies were identified by searching through an extensive range of bibliographic databases from their inception to July 2005 (supplemental table 1). We attempted to identify further studies by contacting clinical experts and by examining the reference lists of all full publication articles retrieved. Unpublished information on current practice and audit data were sought by directly contacting all Primary Care Trust (PCT) leads in child health/community paediatrics and all Strategic Health Authority (StHA) leads in child health services in England and Wales. Published and unpublished studies in any language were eligible for inclusion. The full search strategy is available from the authors on request.

Study selection, data extraction and quality assessment were performed by one reviewer, using standardised forms, and checked by a second. A review-specific tool was developed to assess the methodological quality of studies reporting a "diagnostic yield" on the basis of the number of children screened, the number found to be below/above a threshold for height and the number subsequently diagnosed with a heightrelated condition. In this type of study, only short or tall children are followed up, and therefore complete diagnostic accuracy data are not available. The methodological tool and results of the quality assessment are reported in supplemental table 2 .

The number of cases of all conditions detected (new, existing or unclear) and reported in the included studies was extracted. Detection rates of new cases were calculated separately for the height-related conditions detailed above and for all new cases of any condition that can present with short stature.

Diagnostic yield data could not be pooled using meta-analytic techniques owing to heterogeneity of ages screened and charts and thresholds used. Studies were, therefore, combined in a narrative synthesis.

\section{Economic modelling}

The economic modelling aimed to find the most cost-effective approach to height screening and the diagnosis and treatment of underlying causes of short stature. Raw data from 10 diagnostic yield studies, for new cases detected, were pooled to provide probability distributions for each included condition. Effectiveness data for treatments of the underlying target conditions were obtained from supplementary searches of the literature.

Available data were used to model lifetime costs and outcomes following, as close as was feasible, the NICE guidelines for economic modelling. ${ }^{16}$ An NHS perspective was adopted. Screening, referral and treatment costs were included in the analysis. Cost data were derived from the literature, ${ }^{17-19}$ Department of Health Reference Costs $^{20}$ and UK Social Services. ${ }^{21}$ Costs were reflated to 2006 values, using the Consumer Price Index from the Personal and Social Services Resource Use (PSSRU) data set. ${ }^{22}$ A discount rate of $3.5 \%$ was used when necessary. ${ }^{23}$

A cost-utility (cost/OALY) analysis was conducted; a OALY represents a year of life, adjusted for its quality or perceived value. The model compared two strategies: screening for short stature at entry to school (age 5) with no screening. Tall-stature conditions were excluded owing to their low prevalence and wide variation in diagnosis and treatment. In the screening strategy, referred patients were assumed to be diagnosed and treated early according to their underlying condition. In the no screening strategy, short-stature cases were assumed to be identified later through visits to the GP. The underlying assumption of the model, therefore, is that those found early will receive appropriate treatment with associated gain in OALYs compared with those found later through normal clinical practice. Full details of the modelling are reported elsewhere. ${ }^{24}$

OALY estimates were derived from the literature 2526 and augmented by estimates from the expert clinical panel advising the systematic review. Early detection and treatment were assumed to provide, over a lifetime, five additional OALYs for GHD, TS and psychosocial conditions, and 2.5 additional OALYs for ISS and JH. No screening (with late detection and treatment) was assumed to provide half the OALY gains, that is, 2.5 years for GHD, TS and psychosocial conditions, and 1.25 for ISS and JH. In the sensitivity analysis, all OALY gains were varied to reflect uncertainty in the estimates.

TreeAge Professional 2005 (TreeAge Software Inc, Williamstown, MA) and MS Excel were used for the model. The model was run using a hypothetical cohort of 594000 children, which represents the number of 5-year-old children in England and Wales ${ }^{27}$ to assess the overall effect on costs and benefits. Probabilistic sensitivity analysis was conducted to evaluate uncertainty in the model's estimates.

\section{RESULTS}

\section{Effectiveness review}

Figure 1 shows the flow of studies through the review process and the number of studies excluded at each stage.

Twelve studies described a screening programme that aimed to identify height-related conditions and provided a diagnostic yield of new cases. ${ }^{18}{ }^{28-38}$ Eight studies were conducted in the UK, ${ }^{18} 280^{32-35} 37$ one in Sweden, ${ }^{36}$ one in Spain, ${ }^{29}$ one in Germany $^{38}$ and one in the United States. ${ }^{31}$ Only two studies ${ }^{36} 38$ included conditions relating to tall stature, the remainder aimed to identify conditions of short stature only. A brief overview of the programmes reported and their protocols is given in tables 1 and 2 .

The number of children measured ranged from $1592^{18}$ to $114881 .^{31}$ The percentage of eligible children measured ranged from $45 \%{ }^{29}$ to $90 \%,{ }^{28}$ where reported. The majority of the screening programmes were based on a single screen of the 
Figure 1 Chart showing the flow of studies through the review process.

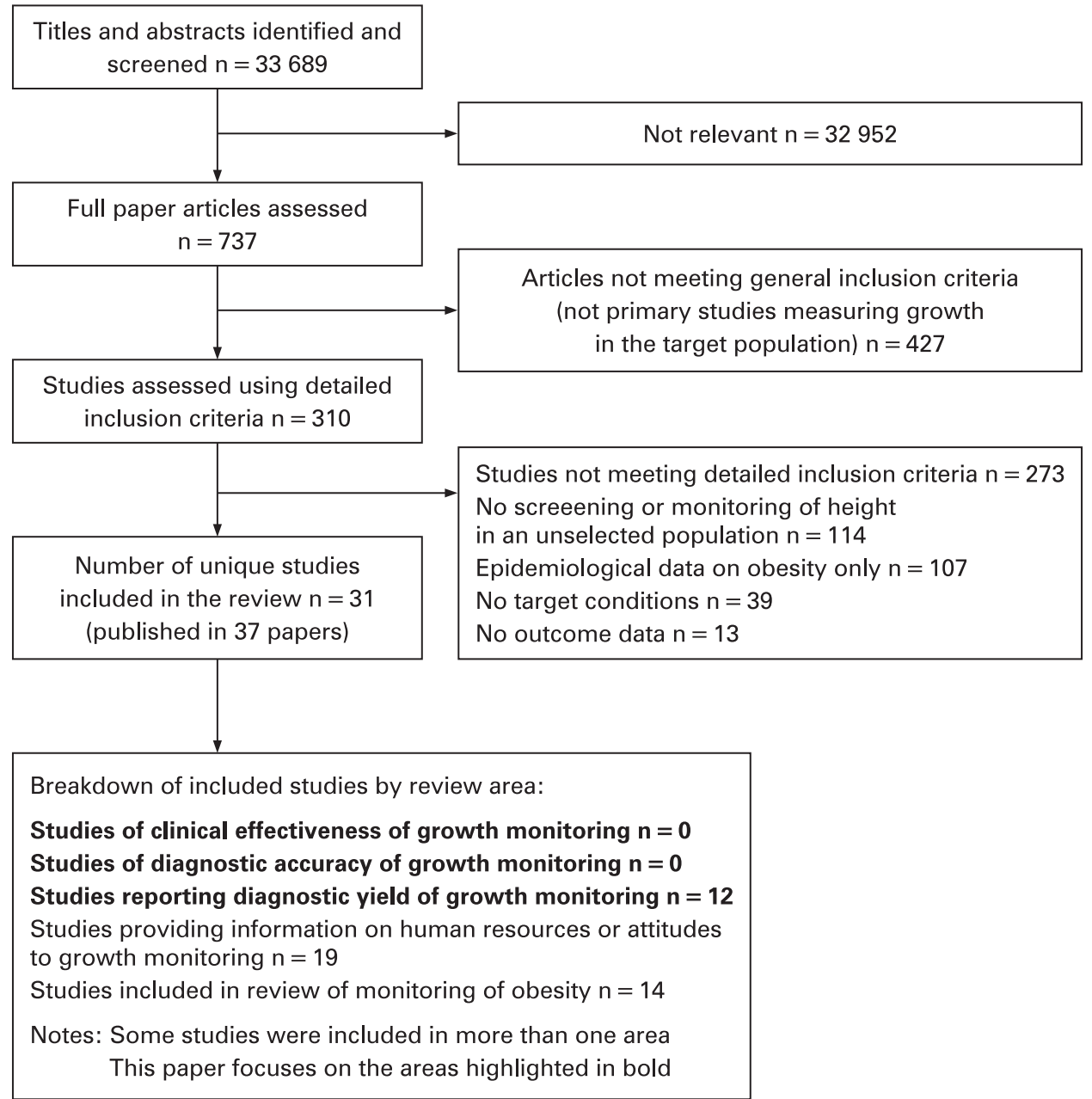

children's height to detect height-related conditions. Five studies measured children of the same age group ${ }^{18} 28323435$ whereas seven studied a cross section of ages using single or serial measurement, but providing results only for the entire cohort rather than by age group. ${ }^{29-31} 3336-38$ Three studies described programmes measuring children at primary-school entry only (ages 4-5) ${ }^{28} 3234$ One study measured children at age 3 and 4.5 years. ${ }^{30}$ Five other studies described screening programmes involving children of primaryschool age (but not restricted to school entry). ${ }^{18} 29313335$ Two studies included a group of older children as well as those at primary school. ${ }^{36}{ }^{37}$ The study conducted in Germany ${ }^{38}$ differed from the others in that it involved a national network of paediatric practices and a computerised monitoring system into which routine height measurements for all children aged 0 to 18 were input on an ongoing basis.

Collectively, the studies' strengths were in reporting a clearly defined selection procedure, providing details of eligibility for the study and indicating whether the sample was random or a whole cohort based on age group and/or region. A description of a reproducible protocol for taking and interpreting height measurements was provided by the majority of the studies. However, almost half of the studies failed to measure $>80 \%$ of the study sample. Three further studies did not explicitly state the number of eligible children, only the number measured; it therefore was not possible to assess their level of coverage. Full quality assessment of all the included studies is provided in supplemental table 2. In addition to the methodological issues detailed in the table, four studies did not have a sufficiently large sample size, given the estimated prevalence of the height-related conditions under consideration, to detect one case of a target condition. ${ }^{18} 282935$

Table 3 lists the number of new cases detected, as reported by each study. Where reported, yield for newly diagnosed GHD (per 1000 children screened) ranged from $0.05^{30}$ to $0.62 .{ }^{38}$ Where reported, for newly diagnosed Turner syndrome yield (per 1000 children screened) was between $0.02^{33}$ and $0.07 .{ }^{38}$ As a secondary gain, height screening identified children with other potentially treatable conditions with a range of detection rates (per 1000 children screened) from $0.22^{31}$ to $1.84 .^{38}$ Three studies did not detect any new cases of any conditions ${ }^{18} 3436$ but all of these had important methodological limitations such as small sample size or high attrition rates at follow-up.

Owing to limitations in the design of included studies, the age or ages at which screening might be most beneficial were unclear. However, the larger, more robust studies of school-entry screening indicated that a single measure at this age might identify new cases at a rate of between $0.54^{30}$ and $0.56^{32}$ per 1000 children screened. It was unclear from the studies that included older age groups what might be the incremental gain from further screening. The diagnostic yield of two of the larger studies of primary-school-aged children may not be reliable as it was unclear whether a number of cases were new or previously diagnosed. ${ }^{31} 33$

\section{Economic modelling}

The incremental cost-utility of height screening at school entry compared with no screening was $£ 9900$ per OALY. This is well 


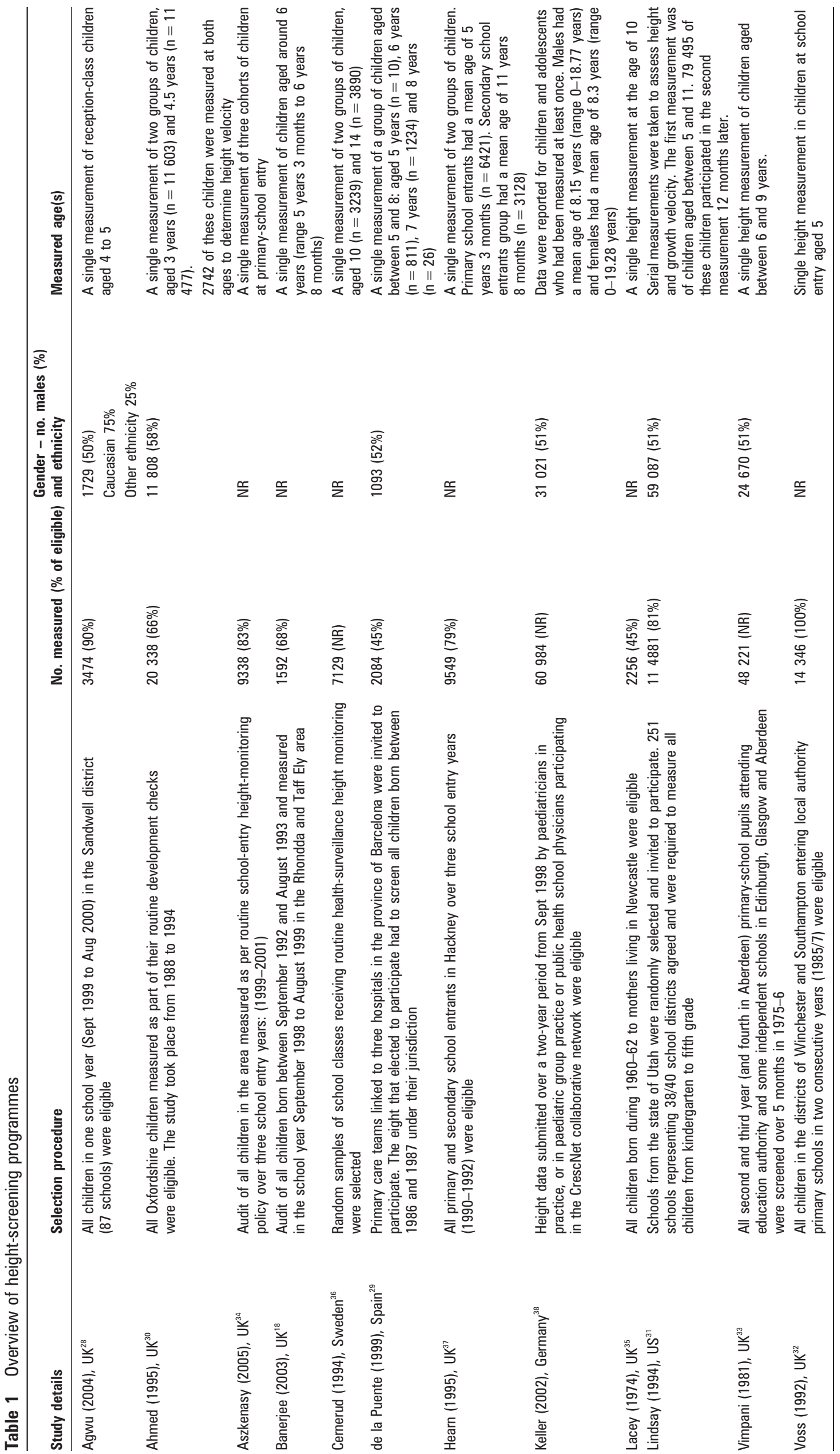


Table 2 Overview of growth-measurement protocols used in included studies

\begin{tabular}{|c|c|c|c|c|}
\hline Study details & Measurer & Method of measuring height & Method of referencing height & Diagnostic threshold \\
\hline Agwu $(2004)^{28}$ & $\begin{array}{l}\text { School nurse, SHN support } \\
\text { worker }\end{array}$ & Leicester height measure & UK 1990 charts & $<0.4$ th centile \\
\hline Ahmed $(1995)^{30}$ & Health visitor & $\begin{array}{l}\text { Microtoise, Minimetre (Raven), } \\
\text { Oxford screening wall charts } \\
\text { (Castlemead Publications) }\end{array}$ & Tanner and Whitehouse 1965 & $\begin{array}{l}\text { (1) }<-2 \text { SDS (referral to auxologist) } \\
\text { (2) }<-3 \text { SDS (referral to consultant) } \\
\text { (3) SDS decrease between } \\
\text { measurements (referral to auxologist) }\end{array}$ \\
\hline Aszkenasy $(2005)^{34}$ & School nurse & Leicester height measure & $\begin{array}{l}\text { 0.4th centile tables based on } \\
\text { UK } 1990 \text { charts }\end{array}$ & $<0.4$ th centile \\
\hline Banerjee $(2003)^{18}$ & $\begin{array}{l}\text { Classroom assistant, } \\
\text { health visitor }\end{array}$ & Leicester height measure & UK 1990 charts & $<0.4$ th centile \\
\hline Cernerud $(1994)^{36}$ & School nurse & $\begin{array}{l}\text { Fixed vertical backboard with } \\
\text { scale and horizonal blade }\end{array}$ & $\begin{array}{l}\text { Reference data used by schools } \\
\text { in the region (unspecified) }\end{array}$ & $\begin{array}{l}\text { Height or height for weight outside } \\
2 \text { SDS, or change of }>0.5 \text { SDS/year }\end{array}$ \\
\hline de la Puente $(1999)^{29}$ & NR & Accustat wall-mounted stadiometer & Growth charts for Catalonia & Less than or equal to the 3 rd centile \\
\hline Hearn $(1995)^{37}$ & School nurse & Minimeter (Raven) & Tanner and Whitehouse, 1965 & $<3$ rd centile \\
\hline Keller $(2002)^{38}$ & Practising paediatrician & Standiometer (Dr Keller system) & German synthetic norm curve & $<3$ rd centile or $>97$ th centile \\
\hline Lacey $(1974)^{35}$ & School nurse & NR & Tanner and Whitehouse, 1965 & $\begin{array}{l}\text { 1st cohort: }<3 \text { rd centile } \\
\text { 2nd cohort: }<-3 \text { SDS }\end{array}$ \\
\hline Lindsay $(1994)^{31}$ & Trained volunteer & Accustat stadiometer & NR & $\begin{array}{l}\text { 1st phase: }<-2 \text { SDS } \\
\text { 2nd phase: }<3 \text { rd centile and growth } \\
\text { rate }<5 \mathrm{~cm} / \text { year }\end{array}$ \\
\hline Vimpani $(1981)^{33}$ & Investigator & Harpenden portable anthropometer & Tanner and Whitehouse, 1965 & $\leqslant-2.5 \mathrm{SDS}$ \\
\hline Voss $(1992)^{32}$ & School nurse & Portable wall-mounted microtoise & Tannerand Whitehouse, 1965 & $<3$ rd centile \\
\hline
\end{tabular}

SDS, standard deviation scores.

within accepted willingness to pay thresholds for the UK (usually £20-30 000 per OALY). Probabilistic sensitivity analysis indicated that the screening programme was cost-effective over $100 \%$ of the model's data distributions for a willingness to pay threshold of $£ 30000$ per QALY. In other words, all projections of the model produced incremental cost per OALY values under $£ 30000$. The majority of costs were incurred in referrals and treatment.

\section{DISCUSSION}

\section{Statement of principal findings}

This review has shown the clinical utility of height screening in terms of increased detection of height-related disorders in children between the ages of 4 and 11 years. We found an incremental yield of between 0.22 and 1.84 per 1000 children screened of undiagnosed treatable conditions including GHD, Turner syndrome, juvenile hypothyroidism and psychosocial growth failure, amongst others. School-entry studies suggested a new case yield of between 0.54 and 0.56 per 1000 children screened.

Reasons for the differences in yield between studies were not clear. The age at which the children were measured is, of course, a significant factor, but differences in yield could also be due to methodological limitations in the study, features of the sample measured or unknown contextual factors (such as previous/ existing monitoring/screening not described in the study).

Economic modelling indicated that height screening is costeffective according to accepted willingness to pay thresholds in the UK of $£ 20-30000$ per OALY.

\section{Strengths and weaknesses of the study}

This is, to our knowledge, the first systematic review to specifically address the effectiveness of height screening to detect height-related conditions in primary-school-aged children. We have used rigorous systematic review methodology to address an important aspect of child health. The review

Table 3 Detection of new cases

\begin{tabular}{|c|c|c|c|c|c|c|c|}
\hline \multirow[b]{2}{*}{ Study } & \multirow[b]{2}{*}{$\begin{array}{l}\text { Age of children } \\
\text { (years) }\end{array}$} & \multirow[b]{2}{*}{$\begin{array}{l}\text { No. children } \\
\text { screened }\end{array}$} & \multicolumn{5}{|c|}{ No. of new cases (yield per 1000) } \\
\hline & & & $\begin{array}{l}\text { Growth hormone } \\
\text { deficiency }\end{array}$ & Turner syndrome & $\begin{array}{l}\text { Juvenile } \\
\text { hypothyroidism }\end{array}$ & $\begin{array}{l}\text { Psychosocial short } \\
\text { stature }\end{array}$ & All conditions \\
\hline Agwu $2004^{28}$ & $4-5$ & 3474 & $2(0.58)$ & & $1(0.29)$ & $1(0.29)$ & $4(1.15)$ \\
\hline Aszkenasy $2005^{34}$ & $4-5$ & 9338 & & & & & \\
\hline Banerjee $2003^{18}$ & 6 & 1592 & & & & & \\
\hline Cernerud $1994^{36}$ & 10,14 & 7129 & & & & & \\
\hline Keller $2002^{38}$ & $0-19$ & 60984 & $38(0.62)$ & $4(0.07)$ & $2(0.03)$ & $3(0.05)$ & $112(1.84)$ \\
\hline Lacey $1974^{35}$ & 10 & 2256 & $1(0.44)$ & & & $1(0.44)$ & $4(1.77)$ \\
\hline Lindsay $1994^{31}$ & $5-11$ & 114881 & $16(0.14)$ & $6(0.05)$ & $3(0.03)$ & & $25(0.22)$ \\
\hline Vimpani $1981^{33}$ & $6-9$ & 48221 & $9(0.19)$ & $1(0.02)$ & $1(0.02)$ & & $11(0.23)$ \\
\hline Voss $1992^{32}$ & 5 & 14346 & $1(0.07)$ & & $1(0.07)$ & & $8(0.56)$ \\
\hline
\end{tabular}

\footnotetext{
*1 in 4000 UK 9 year olds. $\uparrow 1$ in 2500 live female births. $\$ 1$ in 1450 people under the age of 22 in UK. §Unknown.
} 
included a comprehensive search strategy and extensive attempts to obtain unpublished studies. However, it remains possible that we have not identified all relevant height-screening programmes given that some may not have been formally evaluated.

This review was unable to fully define the role of heightscreening programmes in terms of the effectiveness and costeffectiveness of various measurement strategies, owing to weaknesses in the available evidence. Firstly, and most significantly, no controlled studies were found evaluating height screening versus no height screening for the detection of heightrelated conditions. Secondly, no studies were found that reported on the diagnostic accuracy of height-screening programmes for the identification of height-related conditions. The review was based on studies of diagnostic yield that only followed-up children found to be outside the normal height range. Such studies are incomplete in that they only present true positive (number of cases identified) and false positive (number of unnecessary referrals) results. Children found to be of normal height were not followed up, so false negatives (number of cases missed by screening) and true negatives cannot be determined. The available diagnostic yield studies were heterogeneous in terms of the age of participants at screening, methods of measurement and thresholds used for referral. The majority of studies were based on one-off screening for height disorders so the relative merits of serial monitoring strategies cannot be determined. In addition, the majority of studies had further methodological limitations that might affect the reliability of their diagnostic yield estimates: failure to measure $>80 \%$ of their sample and insufficient sample size, given the estimated prevalence, to detect one case of a heightrelated condition.

Although the findings of the economic modelling suggest that height screening is justifiable, the analysis was not able to capture any potential harmful effects of screening associated with negative labelling and inappropriate further referral. Data used to populate the model had a number of additional limitations and therefore the results need to be treated with caution. In particular, there is considerable uncertainty associated with the estimates used to derive OALY gains for early versus late detection and treatment, as these were based on previously published estimates from only two studies and expert opinion. OALY gains used in the model attempted to reflect the increases in health-related quality of life as a result of increases in final height. Data were not available to capture all quality of life gains as a result of treatment. However, although these limitations exist it should be noted that the growthhormone-related OALY gains in a previous study, ${ }^{25}$ used to inform the present model, were given cautious acceptance by NICE in the compilation of guidelines for growth-hormone treatment in children. ${ }^{39}$ Sensitivity analyses considered this uncertainty by attaching a plausible distribution to OALY gains, which is reflected in the results of the model. Full details of the methods and results of the modelling are provided in the published HTA report. ${ }^{24}$

\section{Unanswered questions and future research}

This review strongly points to the need for further research. The role of height screening, alone or in conjunction with other child-health surveillance, needs to be fully defined. Large-scale, long-term controlled trials are needed to determine the optimum strategy. Such trials should evaluate overall benefits and harms alongside costs. Diagnostic accuracy studies could address the relative diagnostic performance of different

\section{What is already known on this topic}

- Height screening has the potential to identify height-related conditions such as growth-hormone deficiency and Turner syndrome, which may present with short stature.

- Early identification of height-related conditions is beneficial.

\section{What this study adds}

- Height-screening programmes in primary-school-aged children are likely to be cost-effective and to give an additional yield of undiagnosed height-related conditions. They can lead to additional detection of other undiagnosed conditions for which short stature is a secondary presentation.

- Further research is needed to determine health utility gains and costs. There is a need for controlled studies to evaluate the exact role of height-screening programmes in improving child health and appropriate measurement strategies to adopt.

screening strategies for the identification of height-related disorders. However, long-term follow-up of both short and normal children would be required to derive sensitivity and specificity estimates and to improve the validity and methodology of future economic-modelling studies.

In future, research should report all factors that might affect detection rates. Studies should clearly report the following details: selection criteria for participants, attempts to contact those eligible, methods used to ensure and check the competence of measurers, a reproducible protocol to ensure consistency of measurements, coverage, measurement error, measurement and follow-up results for all participants. Empirical studies of quality of life gains associated with the early detection and treatment of height-related conditions are warranted to improve long-term cost-utility estimates.

The research described would lend support to the findings of this review and would clarify the role of height screening in improving child health.

Funding: The study was funded by the NHS R \& D under its HTA programme but the authors' work was conducted independently of the funders.

Competing interests: GB has previously received educational and research funding from all the major manufacturers of growth hormone. All other authors have nothing to declare.

\section{REFERENCES}

1. Lee SG. Does child health surveillance contribute to the detection of growth problems? A study of children referred to the growth clinic in Leeds [Master of Medical Science dissertation]. Leeds: University of Leeds, 1996.

2. Aynsley-Green A, Macfarlane JA. Method for the earlier recognition of abnormal stature. Arch Dis Child 1983;58:535-7.

3. Herber SM, Milner RD. When are we diagnosing growth hormone deficiency? Arch Dis Child 1986;61:110-2.

4. Massa G, Verlinde F, De Schepper J, et al. Trends in age at diagnosis of Turner syndrome. Arch Dis Child 2005;90:267-8.

5. Cotterill AM. An international perspective on height surveillance. J Med Screen 1995:2:171-3.

6. Hall DM, Voss LD. Growth monitoring. Arch Dis Child 2000;82:10-5.

7. Job JC. Early diagnosis and early treatment of growth hormone deficiency. Horm Res 1989;31:149-52.

8. Hyer W, Cotterill AM, Savage MO. Common causes of short stature detectable by a height surveillance programme. J Med Screen 1995;2:150-3.

9. Sarr M, Job JC, Chaussain JL, Golse B. Les retards de croissance psycogenes. Etude critique des elements de diagnostic. [Psychogenic growth retardation. Critical study of diagnostic data]. Arch Fr Pediatr 1987;44:331-8. 
10. Centre for Community Child Health Royal Children's Hospital. Child health screening and surveillance: a critical review of the evidence. Melbourne: National Health and Medical Research Council, 2002.

11. National Screening Committee. Child health sub-group report: growth disorders Leeds: National Screening Committee, 2004.

12. Hulse JA, Schilg S. United Kingdom community growth screening 1994: a survey of current practice. J Med Screen 1995:2:154-6.

13. van Buuren S, van Dommelen P, Zandwijken GR, et al. Towards evidence based referral criteria for growth monitoring. Arch Dis Child 2004;89:336-41.

14. de Onis M, Wijnhoven TMA, Onyango AW. Worldwide practices in child growth monitoring. J Pediatr 2004;144:461-5.

15. NHS Centre for Reviews and Dissemination. Undertaking systematic reviews of research on effectiveness: CRD's guidance for those carrying out or commissioning reviews. 2nd edn. York: NHS Centre for Reviews and Dissemination, 2001.

16. National Institute for Clinical Excellence. Guide to the methods of technology appraisal. London: National Insitute for Clinical Excellence; 2004 http:// www.nice.org.uk/pdf/TAP Methods.pdf.

17. Bryant J, Cave C, Mihaylova B, et al. Clinical effectiveness and cost-effectiveness of growth hormone in children: a systematic review and economic evaluation. Health Technol Assess 2002;6(18):1-168.

18. Banerjee S, Morgan RJ, Rees SA, et al. Height screening at school: ineffective without high standards and adequate resources. Arch Dis Child 2003;88:477-81.

19. Macfarlane A. Should we screen for growth problems in children? The pragmatic answer for 1994-5. J Med Screen 1994;1:50-9.

20. Department of Health. NHS reference costs 2004: NHS trust reference cost index. 2004. http://www.dh.gov.uk/PublicationsAndStatistics/Publications/ PublicationsPolicyAndGuidance/PublicationsPolicyAndGuidanceArticle/fs/ en?CONTENT ID = 4105545\&chk = znAfqu (accessed 29 Jan 2008)

21. Tapsfield R, Collier F. The cost of foster care: investing in our children's future. London: British Association for Adoption and Fostering and The Fostering Network; 2005, http://www.pssru.ac.uk.

22. Personal Social Services Research Unit. PSSRU website. PSSRU; 2006.

23. Birch S, Gafni A. The 'NICE' approach to technology assessment: an economics perspective. Health Care Management Science 2004:7:35-41.

24. Fayter D, Nixon J, Hartley S, et al. A systematic review of the routine monitoring of growth in children of primary school age to identify growth related conditions. Health Technol Assess 2007;11:1-182.
25. Anthony D, Stevens A. Growth hormone in children development and valuation committee Report No 57. Bristol: NHS Executive South West; 1996.

26. Busschbach JJ, Rikken B, Grobbee DE, et al. Quality of life in short adults. Horm Res 1998;49:32-8.

27. Office for National Statistics. Birth statistics: review of the Registrar General on births and patterns of family building in England and Wales, 2004. London: Office for National Statistics, 2005.

28. Agwu JC, Leishenring A, Darnley I. Community growth monitoring in practice. Arch Dis Child 2004;89:394.

29. de la Puente ML, Ibanez L, Marcos MV, et al. Cribado problacional de crecimiento en atencion primaria. Grupo de Trabajo de Atencion Primaria. [Primary care population screening for growth]. Aten Primaria 1999;23:346-51.

30. Ahmed ML, Allen AD, Dunger DB, et al. The Oxford growth study: a district growth surveillance programme 1988-1994. J Med Screen 1995;2:160-3.

31. Lindsay R, Feldkamp M, Harris D, et al. Utah Growth Study: growth standards and the prevalence of growth hormone deficiency. J Pediatr 1994;125:29-35.

32. Voss LD, Mulligan J, Betts PR, et al. Poor growth in school entrants as an index of organic disease: the Wessex Growth Study. BMJ 1992;305:1400-2.

33. Vimpani GV, Vimpani AF, Pocock SJ, et al. Differences in physical characteristics, perinatal histories, and social backgrounds between children with growth hormone deficiency and constitutional short stature. Arch Dis Child 1981;56: 922-8

34. Aszkenasy M. Audit of school entry growth screening. Middlesbrough, UK: Tees and North East Yorkshire NHS Trust, West Lane Hospital

35. Lacey KA, Parkin JM. Causes of short stature. Lancet 1974;1:42-5.

36. Cernerud $\mathbf{L}$, Edding $\mathrm{E}$. The value of measuring height and weight of schoolchildren Paediatr Perinat Epidemiol 1994;8:365-72.

37. Hearn S, Cotterill AM, Majrowski W, et al. Construction of a community height surveillance programme: the Hackney growth initiative. J Med Screen 1995;2:31-4

38. Keller E, Gausche R, Meigen C, et al. Auxological computer based network for early detection of disorders of growth and weight attainment. J Pediatr Endocrinol 2002;15:149-56.

39. National Institute for Clinical Excellence. Guidance on the use of human growth hormone (somatropin) in children with growth failure. London: National Institute for Clinical Excellence (NICE); 2002 Report No.: Technology Appraisal no. 42. Available from: http://www.nice.org.uk/pdf/HGHinChild-42-ALS.pdf.

\section{Keep up to date: sign up for our alerting services}

Find out automatically when an article is published on a specific topic or by a particular author. We can also alert you when an article is cited or if an eLetter or correction is published. You can also choose to be alerted when a new issue is published online [and when we post articles Online First]. Check out the New Content Alerts and Citation tracker from the Online tools section on the home page. 